\title{
More than just a metabolic regulator - elucidation and validation of new targets of PdhR in Escherichia coli
}

\author{
Anna-Katharina Göhler ${ }^{1}$, Öznur Kökpinar ${ }^{2,3}$, Wolfgang Schmidt-Heck ${ }^{4}$, Robert Geffers ${ }^{5}$, Reinhard Guthke ${ }^{4}$, \\ Ursula Rinas ${ }^{2,3}$, Stefan Schuster ${ }^{6}$, Knut Jahreis ${ }^{1}$ and Christoph Kaleta ${ }^{6,7^{*}}$
}

\begin{abstract}
Background: The pyruvate dehydrogenase regulator protein (PdhR) of Escherichia coli acts as a transcriptional regulator in a pyruvate dependent manner to control central metabolic fluxes. However, the complete PdhR regulon has not yet been uncovered. To achieve an extended understanding of its gene regulatory network, we combined large-scale network inference and experimental verification of results obtained by a systems biology approach.
\end{abstract}

Results: 22 new genes contained in two operons controlled by PdhR (previously only 20 regulatory targets in eight operons were known) were identified by analysing a large-scale dataset of $E$. coli from the Many Microbes Microarray Database and novel expression data from a $p d h R$ knockout strain, as well as a PdhR overproducing strain. We identified a regulation of the glycolate utilization operon g/CDEFGBA using chromatin immunoprecipitation and gel shift assays. We show that this regulation could be part of a cross-induction between genes necessary for acetate and pyruvate utilisation controlled through PdhR. Moreover, a link of PdhR regulation to the replication machinery of the cell via control of the transcription of the $d c w$-cluster was verified in experiments. This augments our knowledge of the functions of the PdhR-regulon and demonstrates its central importance for further cellular processes in E. coli.

Conclusions: We extended the PdhR regulon by 22 new genes contained in two operons and validated the regulation of the glcDEFGBA operon for glycolate utilisation and the $d c w$-cluster for cell division proteins experimentally. Our results provide, for the first time, a plausible regulatory link between the nutritional status of the cell and cell replication mediated by PdhR.

\section{Background}

The pyruvate dehydrogenase complex of Escherichia coli is encoded by the operon $p d h R$-aceE-aceF-lpdA. The first gene encodes the pyruvate dehydrogenase complex regulator (PdhR), which functions as a transcriptional regulator in a self-regulatory manner for this operon [1]. The multienzyme complex of the pyruvate dehydrogenase complex consists of 24 subunits of the pyruvate dehydrogenase $(a c e E), 24$ subunits of the dehydrolipoate acetyltransferase $(a c e F)$, and 12 subunits of the dehydrolipoamide dehydrogenase $(l p d A)$. This complex catalyses the formation of

\footnotetext{
* Correspondence: christoph.kaleta@uni-jena.de

${ }^{6}$ Department of Bioinformatics, School of Biology and Pharmaceutics, ErnstAbbe-Platz 2, Friedrich Schiller University of Jena, D-07743 Jena, Germany Full list of author information is available at the end of the article
}

acetyl-CoA from pyruvate, which subsequently enters the TCA cycle [2]. Thereby the complex regulates the metabolic flux at the pyruvate node, which connects glycolysis and the TCA cycle.

The pyruvate dehydrogenase complex regulator (PdhR) belongs to the Gnt family of transcription factors [3] and is regulated by a pyruvate-sensing mechanism [1]. While PdhR represses the transcription of its target genes, the pyruvate-bound state of the regulator is not able to bind DNA. PdhR controls not only the transcription of the multi-enzyme complex of the pyruvate dehydrogenase complex, but also targets the $n d h$ and cyoABCDE operons (genes encoding proteins for electron transport), which leads to the hypothesis that PdhR functions as a master regulator of genes involved in energy production and the

\section{C) Biomed Central}


following terminal electron transport from NADH to oxygen [4]. Furthermore, a connection between central metabolism and iron transport has been described by the regulation of the $f e c A B C D E$ operon (genes for ferric citrate transporter) by PdhR [5]. The tomB-hha operon (antitoxin (TomB)-toxin (Hha)-module) [4] and the genes hemL (glutamate-1-semialdehyde aminotransferase) [4], yfiD (pyruvate formate-lyase subunit) [6], and lipA (lipoate synthase) [7] are also directly controlled by PdhR.

Since PdhR plays an important role in the control of metabolic flux, we aimed to identify further targets of this regulator. For this purpose we set out to identify potential targets of regulation by PdhR in a large-scale microarray dataset of $E$. coli from the Many Microbes Microarray Database [8]. Moreover, we constructed a pdhR-knockout and a PdhR-overproducing strain and studied their transcriptome on a variety of growth media. Following this analysis, we identified four potential binding sites of PdhR. Using chromatin immunoprecipitation (ChIP) in combination with quantitative PCR and gel shift assays we discovered that the glcDEFGBA operon (genes for glycolate utilisation, malate synthase) as well as the mraZW-ftsLImurEF-mraY-murD-ftsW-murGC-ddlB-ftsQAZ-lpxC transcription unit (genes for proteins involved in cell division) are controlled by PdhR. Our results thus further underline the central importance of PdhR for the control of metabolism and its involvement in cell division by providing a link to the nutritional status of the cell. In consequence, we further support the notion that PdhR is an important component of the transcriptional regulatory network of $E$. coli $[9]$.

\section{Results}

The outline of our study is presented in Figure 1.

\section{Construction of a $p d h R$ deletion mutant and investigation of the phenotype on different media}

The deletion mutant LJ110 $\Delta p d h R$ was constructed as described in Methods. After confirming the genotype by PCR, the strains LJ110 and LJ110 $\Delta p d h R$ as well as LJ110/pTM30 and LJ110/pTM30PdhRhis were investigated regarding their phenotypical growth behaviour. For this purpose strains were grown overnight and inoculated into various fresh media. Growth behaviour was observed by measuring the change in optical density. The growth rates $\mu\left[\mathrm{h}^{-1}\right]$ are given in Table 1 .

When grown in complex LB medium, neither the $p d h R$ deletion mutant nor the PdhRhis overexpression strain differed in their growth behaviour from the parental strains. In contrast, when grown in minimal media supplemented with glucose, glycerol, or acetate as carbon source, a severe growth defect of the $p d h R$ deletion mutant was observed. This phenotype emerged regardless of the carbohydrate source. Interestingly, this growth defect did not occur when cells were grown in minimal medium supplemented with pyruvate. Since the transcription factor PdhR is inactivated by pyruvate, the deletion is of no consequence under these conditions. Overproduction of the transcription regulator in minimal medium did not affect growth behaviour, as was observed in rich medium.

To get a more detailed insight into the effect of the chromosomal $p d h R$ deletion and PdhR overproduction on the transcriptome, we analysed the gene-expression profiles of these strains under various growth conditions using microarrays.

\section{Elucidating further targets of PdhR in a systems biology approach}

We used three different approaches to infer further targets of PdhR. Firstly, we identified putative targets of regulation by PdhR through the analysis of a large-scale gene-expression data set from the Many Microbes Microarray Database $\left(\mathrm{M}^{3 D},[8]\right)$.

Secondly, we analysed microarray data from a PdhR overproducing and a $p d h R$ knockout strain. Thirdly, we inferred putative phylogenetically conserved binding sites of PdhR on a genome scale using a previously described approach [7].

In the first approach, we determined an association score (z-score) indicating the significance of a regulation of each gene in the genome of E. coli by PdhR. These scores were determined from 907 gene-expression experiments, stored in $\mathrm{M}^{3 D}$, using the context-likelihood of relatedness algorithm [5]. In the second approach, we obtained a regulatory score that corresponds to the strength of the effect of a knockout as well as an overproduction of PdhR on the expression of each gene during growth on three different media. In the third approach, we searched for putative phylogenetically conserved binding sites of PdhR in the upstream region of each gene of E. coli. We identified putative binding sites in the promoter regions of 363 operons containing 642 genes.

To identify further potential targets of PdhR, we sorted all genes in whose promoter region we identified a putative binding site of PdhR independently according to their z-scores and regulatory scores. The results of this analysis are displayed in Table 2 . Thus, we found that particular genes belonging to the $d c w$ cluster - which is important in cell division [10] - are the top-ranking targets according to their z-score. In contrast, genes of the glcDEFGBA operon, which are important in glyoxylate and glycolate utilisation, are enriched among the targets identified from the regulatory score. In addition to these two operons, we selected $y n f M$ and metB as likely targets of a regulation by PdhR. We selected $y n f M$ since it encodes a putative transport protein belonging to the major facilitory superfamily of transporters [11] and 


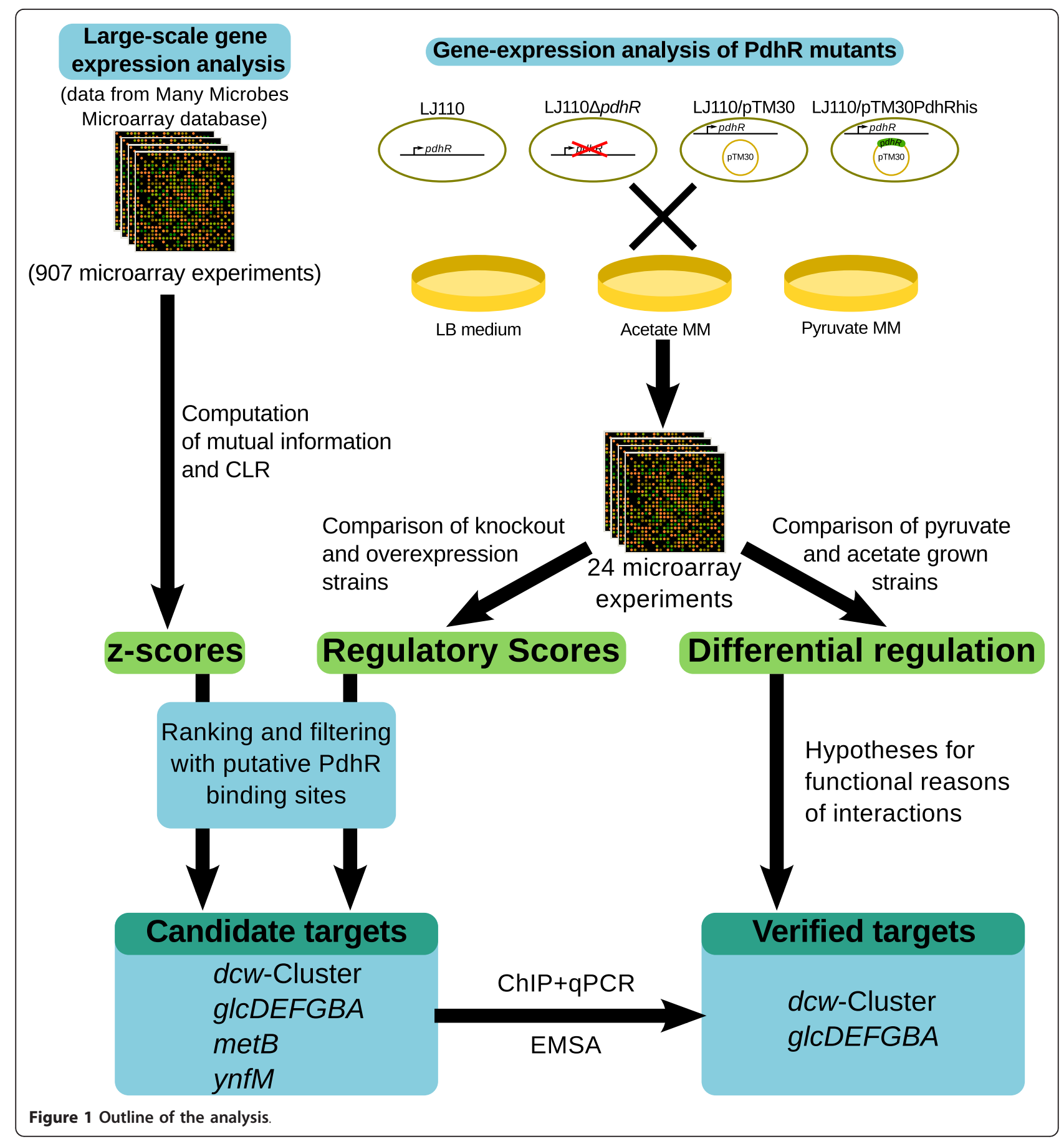

Table 1 Effect of pdhR deletion and overexpression on growth

\begin{tabular}{|c|c|c|c|c|c|}
\hline Strain & LB, $\mu\left[h^{-1}\right]$ & MM glucose $\mu\left[h^{-1}\right]$ & MM glycerol $\mu\left[\mathrm{h}^{-1}\right]$ & MM acetate $\mu\left[h^{-1}\right]$ & MM pyruvate $\mu\left[h^{-1}\right]$ \\
\hline ப110 & $1.29+/-0.01$ & $0.59+/-0.07$ & $0.42+/-0.01$ & $0.17+/-0.02$ & $0.21+/-0.07$ \\
\hline L110 $\Delta p d h R$ & $1.25+/-0.06$ & $0.20+/-0.019$ & $0.19+/-0.02$ & $0.07+/-0.02$ & $0.22+/-0.00$ \\
\hline L110/pTM30 & $1.28+/-0.02$ & $0.60+/-0.00$ & $0.41+/-0.01$ & $0.14+/-0.03$ & $0.20+/-0.02$ \\
\hline L110/pTM30PdhRhis & $1.26+/-0.01$ & $0.53+/-0.00$ & $0.43+/-0.01$ & $0.13+/-0.03$ & $0.19+/-0.01$ \\
\hline
\end{tabular}

Strains with different genotypes regarding $p d h R$ were grown in rich medium (LB) and minimal medium (MM) supplied with glucose, glycerol, acetate or pyruvate as carbon source. The growth rate was determined in the mid logarithmic phase. 
Table 2 Identification of further targets of PdhR

\begin{tabular}{|c|c|c|c|c|c|c|c|}
\hline \multirow[b]{2}{*}{ ID } & \multicolumn{3}{|c|}{ Targets sorted by z-score } & \multicolumn{4}{|c|}{ Targets sorted by regulatory score } \\
\hline & Gene & Regulatory score & z-score & ID & Gene & Regulatory score & z-score \\
\hline b1109 & $n d h^{*}$ & 2.913 & 7.803 & b1109 & $n d h^{*}$ & 2.913 & 7.803 \\
\hline b0091 & murc $\$$ & -0.030 & 5.958 & b0114 & aceE* $^{*}$ & 2.250 & 5.866 \\
\hline b0115 & $a c e F^{*}$ & 2.008 & 5.883 & b4467 & $g / c F^{\$}$ & 2.181 & 0.275 \\
\hline b0114 & $a c e E^{*}$ & 2.250 & 5.866 & b0115 & aceF* & 2.008 & 5.883 \\
\hline b0088 & murD $\$$ & -0.013 & 5.623 & b2579 & yfiD* & 1.973 & 0.805 \\
\hline b0090 & $\operatorname{murG} \$$ & -0.015 & 4.773 & b2979 & $g / C D^{\$}$ & 1.853 & 1.709 \\
\hline b0089 & $\mathrm{fts} W^{\lessgtr}$ & 0.073 & 4.664 & b4467 & $g / c F^{5}$ & 1.163 & 0.275 \\
\hline b0084 & $\left.\mathrm{fts}\right|^{\$}$ & 0.140 & 4.556 & b2975 & $g / c A^{\$}$ & 1.144 & 0.400 \\
\hline b0082 & $\mathrm{mraW}^{\$}$ & -0.056 & 4.297 & b2977 & $\mathrm{glcG}^{\$}$ & 1.056 & 2.315 \\
\hline b0087 & mrar & 0.086 & 4.229 & b2601 & arof & 1.051 & 1.723 \\
\hline b0125 & hpt & 0.002 & 4.149 & b3828 & metR & 1.035 & 1.449 \\
\hline b3613 & enve & -0.005 & 3.938 & b2976 & $g / c B^{\$}$ & 0.977 & 2.124 \\
\hline b0628 & $\operatorname{lip} A^{*}$ & -0.075 & 3.914 & b1596 & ynfM & 0.927 & 0.007 \\
\hline b4052 & $d n a B$ & -0.018 & 3.864 & b2600 & tyrA & 0.915 & 2.065 \\
\hline b0085 & mure $E^{\$}$ & 0.167 & 3.659 & b2505 & $y f g H$ & 0.820 & 0.425 \\
\hline b0822 & ybiV & 0.284 & 3.653 & b0333 & prpC & 0.729 & 0.021 \\
\hline b2683 & ygaH & -0.077 & 3.628 & b0331 & $\operatorname{prp} B$ & 0.713 & 0.024 \\
\hline b0436 & tig & -0.260 & 3.620 & b3939 & met $B$ & 0.677 & 2.667 \\
\hline b4290 & $f e c B^{*}$ & 0.315 & 3.561 & b3547 & yhjx & 0.668 & 2.931 \\
\hline b0083 & $\mathrm{fts}^{\mathbf{S}}$ & 0.119 & 3.536 & b3426 & $g / p D$ & 0.659 & 0.904 \\
\hline
\end{tabular}

Top-ranking genes with putative phylogenetically conserved binding sites of PdhR sorted according to z-scores (first four columns) and regulatory scores (last four columns). Previously known targets of PdhR are marked with "** and new targets confirmed in this study with "\$". Additionally to the new experimentally validated targets indicated in the above table, a regulation of $m r a Z$, murF, ddIB, fts $Q$, fts $A$, fts $Z$ and $I p x C$ by PdhR was confirmed as they are part of the operon that forms the $d c w$-cluster.

displays a marked increase of expression during growth on pyruvate, thereby being a potential pyruvate transporter (Additional File 1). Moreover we identified metB, which encodes an enzyme in methionine biosynthesis, as a putative target of PdhR.

\section{Verifying four predicted binding sites by ChIP and qPCR}

The analysis of our DNA microarray data combined with the data from databases, revealed new operons and pathways which might be regulated by PdhR. In the first experimental step four potential binding sites were chosen for further verification.

We tested the putative PdhR binding sites within the operator fragments of the genes $g l c D$ (encodes a subunit of the glycolate oxidase), $m r a Z$ (encodes a conserved protein in front of the $d c w$ cluster), met $B$ (encodes homocysteine transmethylase), and $y n f M$ (encodes an unknown transporter protein) by chromatin immunoprecipitation (ChIP) and quantitative PCR (qPCR). Therefore, a culture which expresses his-tagged PdhR was grown in LB medium. The transcription regulator was cross-linked to chromosomal DNA. After cell lysis and shearing the DNA, DNA-repressor complexes were co-precipitated, the crosslinking reversed, and the DNA purified. This DNA was used as template DNA in the
qPCR to compare the amounts of precipitated DNA fragments containing the described binding sites.

Results are shown in Figure 2. For the tested primer pairs, the amount of PCR product for the ptsG gene that is not regulated by PdhR, served as a negative control PCR and was set as 1. DNA fragments of the known binding target for PdhR self-regulation in front of the $p d h R$ gene were enriched by a factor of 11.6 in the assay. The GPCR revealed an enrichment of DNA fragments which contain the putative binding sites for PdhR in front of the genes $g l c D$ and $m r a Z$ by a factor of 1.84 and 1.72 , respectively, that are statistically significant (Figure 2).

No enrichment was found for the putative PdhR target sequences in front of the genes metB (factor 0.98) and ynfM (factor 0.06). The applied growth conditions in complex rich medium might not be suitable for the detection of all PdhR-DNA interactions, although the overproduction of PdhRhis should facilitate binding, also to DNA fragments which might be bound with low affinity.

Verifying three binding sites in vitro by gel shift assays In a second experimental step, three putative binding sites were further investigated. The binding activity of 


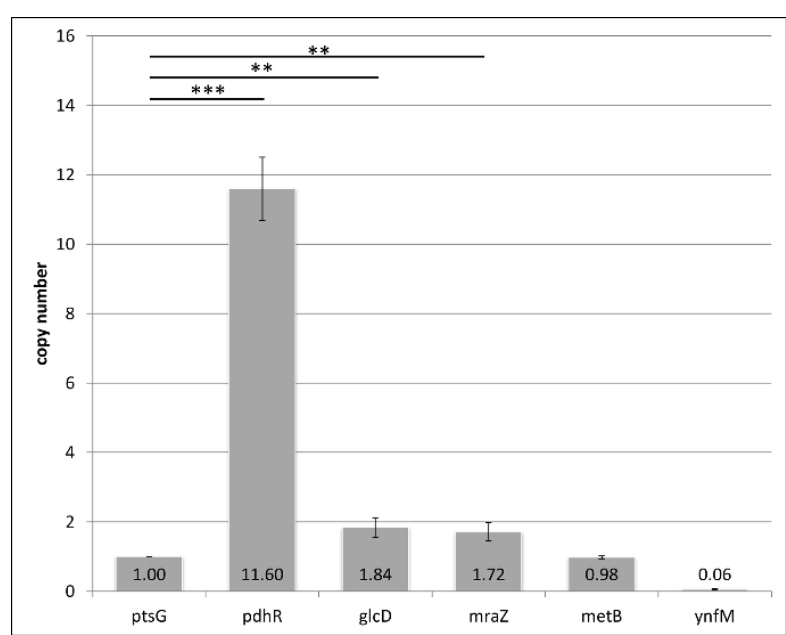

Figure 2 Results of the ChiP and qPCR experiments. His-tagged PdhR was crosslinked to DNA and purified. The co-precipitated DNA was analysed for the frequency of copies, which contain the putative binding sites of PdhR. The operator fragment of the not controlled pts G gene was set as 1. The amount of DNA fragments which contain the known self-regulating pdhR binding site of $P d h R$ was enriched to 11.6 times more copies compared to the negative control. The g/cD and mraZ operator fragments occur to be 1.8 and 1.7 more abundant than the control fragment. The metB DNA fragment was detected with the same frequency as the control fragment and the $y n f M$ binding site was observed to be less precipitated than the negative control. The data are mean values with standard deviations of three experiments. The statistical significance of the binding of PdhR to $p d h R, g / c D$ and $m r a Z$ if compared to the control by a One-Way-ANOVA test is indicated by asterisks (***: p-value $<0.001,{ }^{* *}$ : p-value $\left.<0.01\right)$.

PdhR towards the binding sites in front of the genes $g l c D$, mraZ and metB were also analysed by electrophoretic mobility shift assays. His-tagged PdhR was purified and incubated with fluorescence labelled DNA fragments. A complex formation of DNA and repressor protein leads to an electrophoretic retardation and thus a shifted fluorescence signal of the DNA fragment. The results are shown in Figure 3. The binding of purified PdhR to the known binding site of the operator region in front of the $p d h R$ gene was observed. The same binding activity was detected for the region in front of the $g l c D$ gene, whereas the complex with the predicted binding site in front of the mraZ gene was much weaker. A complex with the DNA binding site of the $m e t B$ gene was not detected in this in vitro approach.

\section{Assessing the global architecture of the PdhR regulon}

Pertaining to the large number of processes regulated by PdhR we furthermore determined its mean expression level over the mean expression levels of all transcription factors of $E$. coli in the microarray data of $\mathrm{M}^{3 D}$. Previously it was found that the number of targets of a transcriptional regulator correlates with its expression

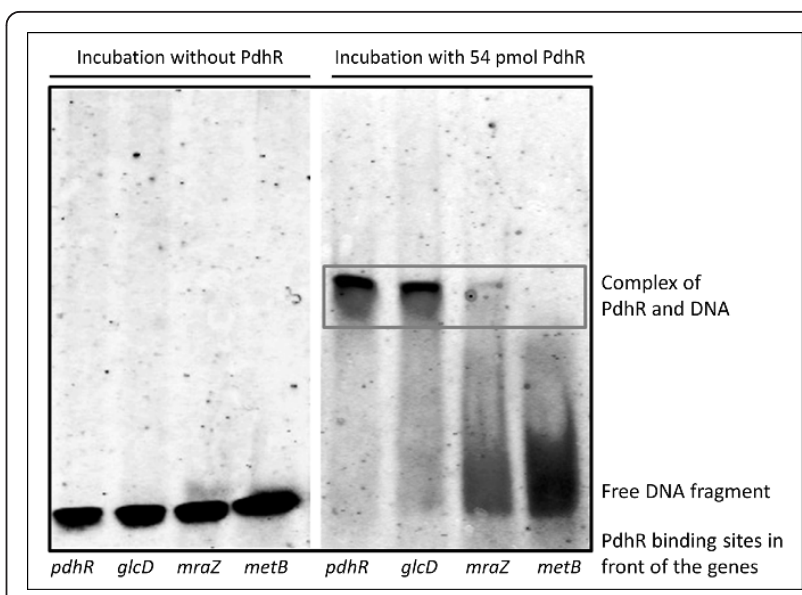

Figure 3 Electrophoretic mobility shift assays. The investigated binding sites in front of the genes $p d h R, g / c D, m r a Z$, and met $B$ are displayed on the lower line. The left hand panel demonstrates an assay for $0.1 \mathrm{pmol}$ of each DNA fragment without PdhR. For the assay shown on the right hand site, samples were incubated with 54 pmol purified PdhR prior to electrophoresis. Complex formation of regulator protein and DNA fragment leads to a shifted DNAsignal, which is assigned by the grey box. A strong PdhR-DNA complex is detected for the binding site in front of the $p d h R$ gene for the known self-regulation. The same binding activity was monitored for the glcD binding site. Very weak affinity of PdhR was detected towards the mraZ binding site, nevertheless a small amount of shifted DNA was observed. No complex formation of PdhR with the binding site in front of metB gene was monitored.

level [12]. The expression level of PdhR is close to the median of expression levels of all transcription factors. This suggests that while controlling distinct key cellular processes, PdhR does so by controlling a selected number of target genes rather than a large number of target genes like other global transcriptional regulators.

\section{Discussion}

\section{Regulation of gICDEFGBA by PdhR}

The $g l c D$ gene is the first gene of the transcription unit glcDEFGBA. This unit encodes the glycolate oxidase (GlcDEF), a small conserved protein of unknown function (GlcG), the malate synthase G (GlcB), and a glycolate transporter protein (GlcA). The operon is activated by $\mathrm{GlcC}$ during growth on glycolate and transcribed as a polycistronic message. The expression also depends on the integrative host factor (IHF) and is repressed by the respiratory regulator ArcA-P [13]. Glycolate and acetate are degraded via the common intermediate glyoxylate. Glyoxylate is an important intermediate of the central metabolism under conditions when acetate or fatty acids are the main carbon and energy source and is metabolised using the so-called glyoxylate bypass [14-16]. There are two isoenzymes, the malate synthases $\mathrm{A}$ and $\mathrm{G}$ (AceB and GlcB) that convert glyoxylate into malate. Both proteins belong to the acetate (AceB, encoded in 
the ace $A B K$ operon) or the glycolate/glyoxylate pathway (GlcB), respectively. Both operons are similarly controlled by the factors IHF and ArcA-P and can fulfil redundant roles via cross-induction [13] to avoid the toxic accumulation of glyoxylate.

The commonly assumed route for pyruvate assimilation proceeds via the phosphoenolpyruvate synthase PpsA which allows gluconeogenesis from this compound. However, in a previous study it was found that the expression level of PpsA is suboptimal for growth on pyruvate [17]. This suggests that alternative routes may exist for pyruvate assimilation. The glyoxylate shunt could serve this purpose as it allows gluconeogenesis from the pyruvate derivative acetyl-CoA. This hypothesis is supported by the observation that the genes for PpsA and the glyoxylate shunt enzymes, AceA and AceB, did not show noticeable differences in expression during growth on acetate and pyruvate (Figure. 4). Moreover, we find that several genes which encode enzymes involved in the conversion of pyruvate into acetate are strongly upregulated in pyruvate grown cultures as compared to acetate grown cultures. Thus, a transcriptional regulation of the malate synthase GlcB, which is part of the $\operatorname{glcDEFGBA}$ operon and also part of the glyoxylate shunt, would allow the cell to control gluconeogenesis from pyruvate via the glyoxylate shunt (Figure. 4).
Extending the results of Pellicer and colleagues [13] who found a cross-induction of genes required for glycolate and acetate assimilation, our findings show that this crossinduction might also extend to genes activated during growth on pyruvate. This is supported by the regulation of the $g l c D E F G B A$ operon by PdhR and the finding, that many genes known to be upregulated during growth on acetate show no marked difference in expression during growth on acetate and pyruvate, while genes which encode enzymes that convert pyruvate into acetate are strongly upregulated during growth on pyruvate but not on acetate.

\section{Regulation of cell division by PdhR}

$\mathrm{MraZ}$ is a protein of unknown function and is encoded in the transcription unit mraZW-ftsLI-murEF-mraY-murD$f t s W$-murGC-ddlB-ftsQAZ-lpxC which represents the $d c w$ cluster [10]. This unit encodes proteins involved in cell division and peptidoglycan biosynthesis. The expression of these genes is regulated in a highly sophisticated manner in order to time the cell division precisely (Figure 5). The gene products of this operon need to be synthesised at the correct time in the correct amount, and cell division has to be prevented when stress conditions prevail. It was shown that the mraZ1p promoter leads to transcription up to the $f t s W$ gene [10]. The whole unit is also postulated to be transcribed in one message from the $m r a Z 1 p$

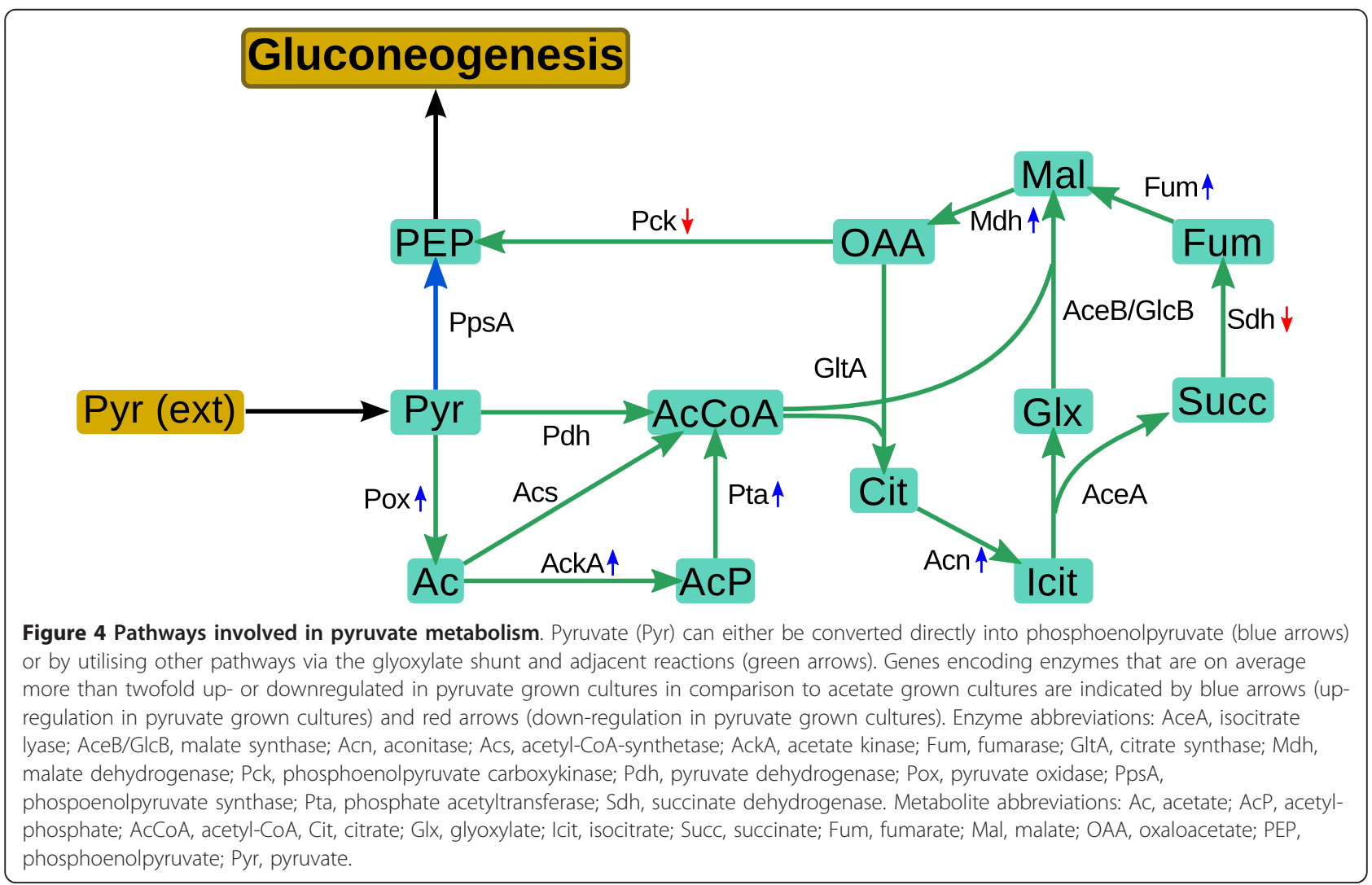




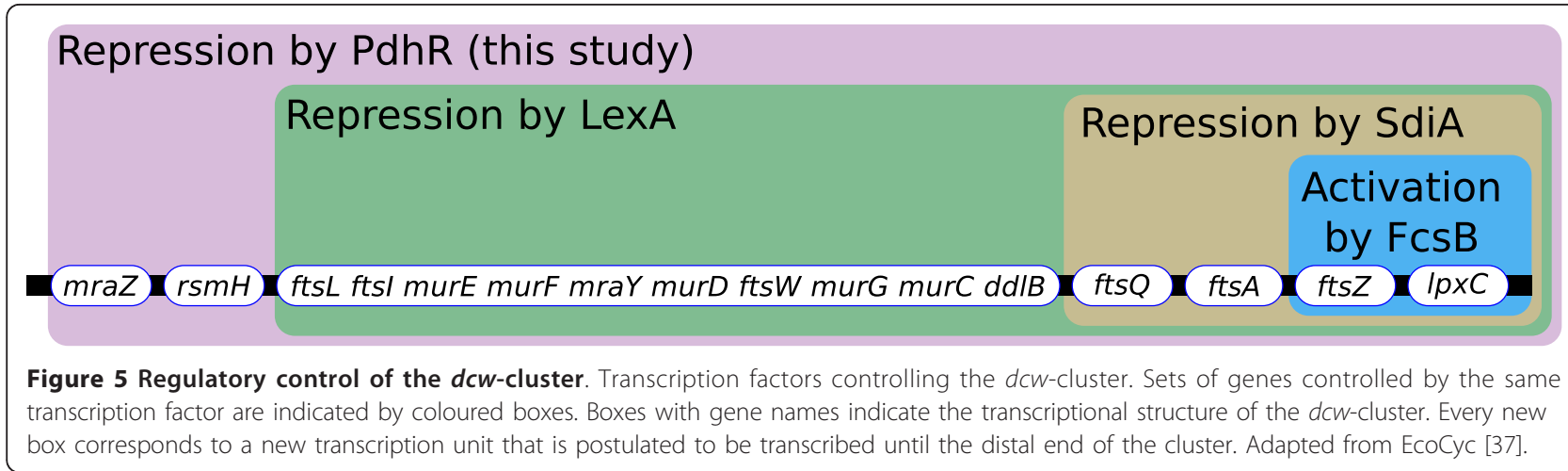

operator to the very distal gene $l p x C$ [10]. Furthermore, there are many other promoter structures described or predicted in the cluster. Five transcription starts can be identified in the 5 ' region of the cluster. This regulation also includes repression by LexA binding to three identified SOS boxes. The transcriptional repressor LexA inhibits expression of genes involved in response to DNA damage and DNA replication inhibition in the so-called SOS response [18]. The repressor is inactivated by RecAdependent cleavage after DNA damage [19].

At the 3' end of the cluster, six promoters have been identified that contribute to the correct timing and concentration of the fts $Z$ gene product which is essential for cell division [20]. Their expression is balanced by the promoters being constantly expressed gearbox promoters. These gearbox promoters are $\sigma^{\mathrm{s}}$ dependent promoters for growth rate regulation, inversely growth rate dependent-, and housekeeping promoters [21]. Additionally, an SdiA (Suppressor of the cell division inhibitor) dependent mechanism is integrated, which couples expression regulation to a quorum-sensing mechanism. Moreover, activation by the phosphorylated RcsB regulator (Regulator capsule synthesis B) was shown.

For normal cell growth and correct division, a balance between the 5' and 3' encoded genes of the $d c w$ cluster is required (for more details on $d c w$ regulation see [10] and references therein).

Even though many regulatory mechanisms for the $d c w$ cluster have already been identified, it is still not known how a link between the metabolic status of the cell and cell division is established. The pyruvate dehydrogenase complex regulator could fulfil this function, since it provides a cue to the nutritional status of the cell by sensing the pyruvate concentration. For instance, during growth on glucose, PdhR acts as a flux sensor for the glycolytic flux [22] that can serve as a proxy for the nutritional status of the cell. In the case of a high glycolytic flux, indicated by higher levels of pyruvate, the negative influence of PdhR on the $d c w$ cluster is reduced whereas it is increased by a low glycolytic flux resulting in low pyruvate levels.

The influence of the metabolic status on cell division by PdhR is reflected by a high z-score in the large-scale analysis of microarray experiments. It is also experimentally supported by the fact that we detected a statistical significant enrichment of the mraZ operator binding site for PdhR by a factor of 1.7 in the in vivo assay. Up to this point we had only been able to detect very weak binding in our in vitro assays. This weak binding in vitro might be due to the complex regulation of the $d c w$ cluster which most likely requires further interacting partners that we could not provide in our in vitro experiments.

\section{Conclusions}

Taken together, we identified 22 new target genes contained in two operons controlled by PdhR using a bioinformatic and an experimental approach. The regulation of the $g l c D E F G B A$ operon and $g l c B$ in particular, as well as the comparison of gene-expression of acetate and pyruvate grown strains demonstrated that the metabolic state of the cell in both conditions is very similar. In particular, we found that in addition to the direct route to gluconeogenesis through the phospoenolpyruvate synthase, alternative pathways for the conversion of pyruvate to acetate appear to be activated and thus provide additional substrates for gluconeogenesis through action of the glyoxylate shunt.

Moreover, we have identified the $d c w$-cluster containing proteins required for cell division as a further regulatory target of PdhR. Through this regulatory interaction, we have established a plausible link between the nutritional status of the cell and cell replication which was not known to date. These results further support the hypothesis that $\mathrm{PdhR}$ is an important regulator of diverse processes of the cell by controlling a selected set of target genes rather than a large number of target genes like other global regulators (Figure 6). Thus, we have also demonstrated that, apart from being a central hub of metabolic fluxes, the 


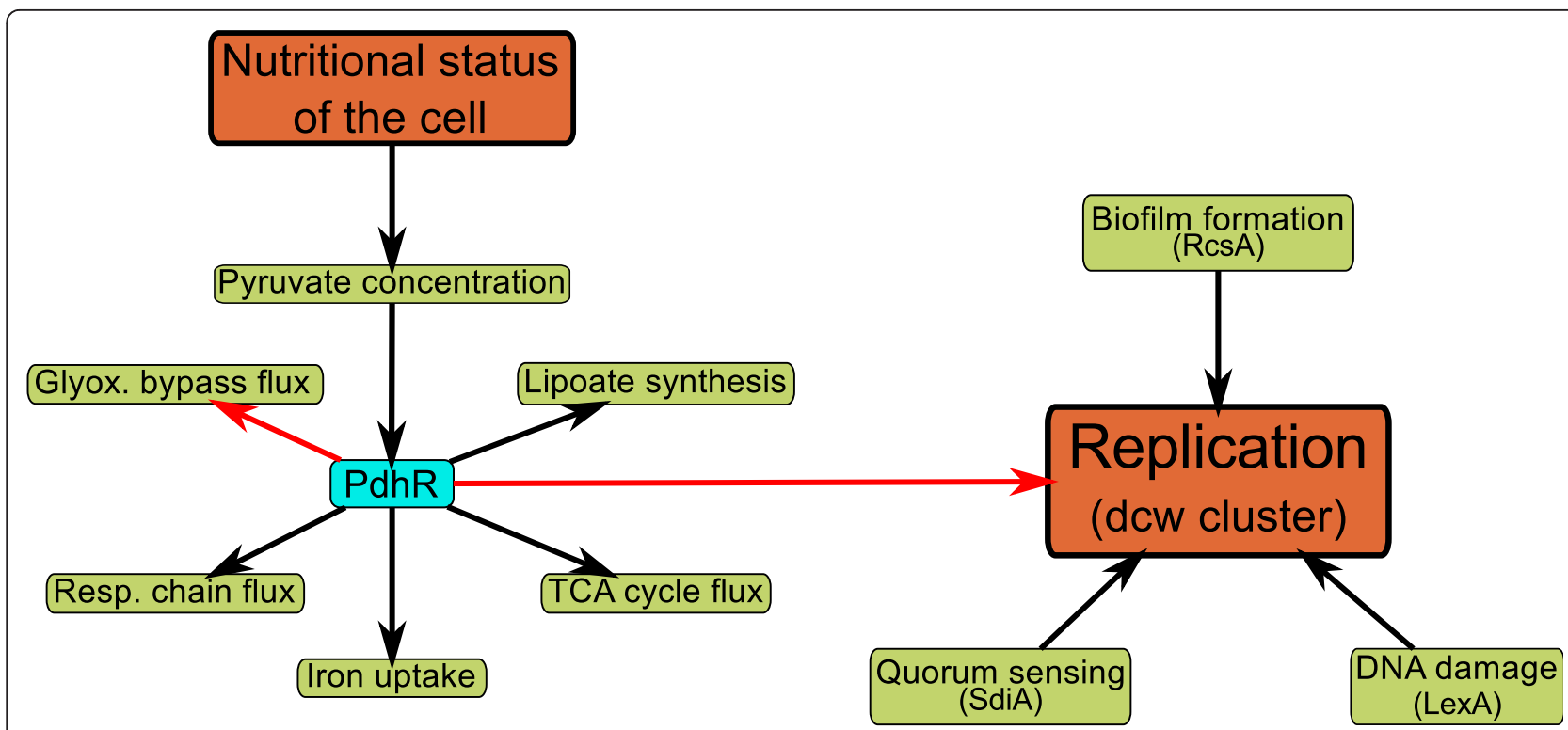

Figure 6 A new view on the PdhR regulon. PdhR acts as an important regulator of many cellular processes. The newly discovered regulation of the $d c w$-cluster by PdhR moreover provides an avenue through which the nutritional status of the cell can influence the replication process. Interactions drawn in red have been identified in this work.

pyruvate node exerts control on many aspects of bacterial physiology. In consequence, PdhR represents a promising target for further studies aimed at understanding central aspects of the interplay between metabolism and other cellular processes.

\section{Methods}

\section{Experimental procedures}

Bacterial strains, plasmids and media

The Escherichia coli K-12 strains LJ110 [23] and LJ110 $\Delta p d h R$ (this study) were grown in Luria-Bertani broth (LB) or standard phosphate minimal medium [24] supplemented with $0.2 \%$ carbon source. When carrying the plasmids pTM30 [25] or pTM30PdhRhis [7], ampicillin was added in a concentration of $50 \mathrm{mg} /$ liter. Cells were incubated at $37^{\circ} \mathrm{C}$ with shaking.

For overproduction of his-tagged PdhR the strain JM109 [26] was transformed with pTM30PdhRhis and gene expression induced with $1 \mathrm{mM}$ IPTG. For construction of a $p d h R$ deletion mutant, the strain BW25113 and the plasmids pKD4 and pKD46 [27] were used. If necessary, kanamycin was added in a concentration of $25 \mathrm{mg} / \mathrm{liter}$.

For the deletion of the $p d h R$ gene we followed the protocol of Datsenko and Wanner [27]. A 1728 bp PCR product was generated by standard PCR with the primer pair Pdhr_wanner+ (ATCCGCCAACCAAAACTCTCCGATG TGATTGAGCAG CAACTGGGTGTAGGCTGGAGC TGC) and Pdhr_wanner- (TTTCGTTGCTCCAGACGAC GCAGAGAACGCTCA CGGCGGCTCTCTTCACGCATATGAATATCCTCCTTAG) and the plasmid pKD4 as template. The PCR product containing the kanamycin resistance cassette with flanking regions that are homologous to chromosomal sequences at the 5' and 3 ' end of the $p d h R$ gene was purified with the Wizard DNA purification system (Promega), DpnI treated and further enriched by ethanol precipitation. In the next step it was transformed into BW25113 carrying pKD46. Transformants grown on LB plates with kanamycin were picked and the successful integration of the resistance cassette was verified by PCR using different primer combinations of $\mathrm{k} 1$ (CAGTCATAGCCGAATAGCCT), k2 (CGGTGC CCTGAATGAACTGC), kt (CGGCCACAGTCGATGAATCC), pdhr_downstream (TGATTTACAACATCTTCTGG) and pdhr_upstream (TGACTTCGGCAAGTGGCTTAAGAC). The chromosomal deletion of the $p d h R$ gene in the BW25113 strain was then transduced into the strain LJ110 via P1 vir transduction generating the strain LJ110 $\Delta p d h R$ following protocols by Arber [28] and Lengeler [29].

\section{Determination of the growth rate}

To determine the growth rate $\mu$, cultures were grown overnight. The next day $10 \mathrm{ml}$ medium was inoculated to an optical density ${ }_{650 / 420}=0.1$ and the OD was measured every hour. The growth rate $\mu$ was determined during the mid-logarithmic phase.

\section{Sample preparation for microarray analysis}

Cells were grown in LB- or minimal medium containing appropriate carbon sources and antibiotics. $1 \times 10^{9}$ cells (LB medium: $\mathrm{OD}_{600}=1,1 \mathrm{ml}$ culture; minimal medium: $\mathrm{OD}_{420}=0.5,4 \mathrm{ml}$ culture) were directly added to two volumes of RNAprotect Bacteria Reagent (Qiagen), mixed by shaking and incubated for $5 \mathrm{~min}$ at room temperature. Cells were pelleted by centrifugation $(5,000 \mathrm{rpm}, 10 \mathrm{~min})$, 
the supernatant was removed and the pellet stored at $-80^{\circ} \mathrm{C}$.

\section{DNA microarray hybridization and analysis}

Total RNA was isolated from the cells using the protocol accompanying the RNeasy Mini Kit (Qiagen; Hilden, Germany). Quality and integrity of the total RNA was controlled on an Agilent Technologies 2100 Bioanalyzer (Agilent Technologies; Waldbronn, Germany). 200 ng of total RNA were applied for Cy3-labelling reaction using the MessageAmp II-Bacteria Kit according to supplier's recommendation (Ambion; Kaufungen, Germany). As a result of IVT (in vitro transcription) reaction using aminoallyl-dUTP antisense aRNA were generated and subsequently coupled with fluorescent dye Cy3. Cy3-labeled aRNA was hybridized to Agilent's $8 \times 15 \mathrm{k}$ E. coli microarray (Agilent Technologies; Waldbronn, Germany, AMADID 020097) for $16 \mathrm{~h}$ at $68^{\circ} \mathrm{C}$ and scanned using the Agilent DNA Microarray Scanner. Expression values (raw data) were calculated by the software package Feature Extraction 10.5.1.1 (Agilent Technologies; Waldbronn, Germany) using default values for GE1_105_Dec08 extraction protocol. Further data manipulation was applied according to section "Analysis of expression data from $p d h R$ knockout and overexpressing strains». Gene-expression data has been submitted to Gene Expression Omnibus under the accession number GSE31333.

\section{Chromatin immunoprecipitation}

Chromatin immunoprecipitation based on a protocol from Jeremiah Faith (http://www.jeremiahfaith.com/open_notebook_science/ C.3 ChIP Protocols and [5]) was adapted with changes. $50 \mathrm{ml}$ of LB medium in a $500 \mathrm{ml}$ flask were inoculated with an overnight culture of LJ110/ pTM30PdhRhis to an $\mathrm{OD}_{650}=0.1$ and grown for $30 \mathrm{~min}$. The expression of the transcription factor was induced with $100 \mu \mathrm{M}$ IPTG. At an $\mathrm{OD}_{600}=115 \mathrm{ml}$ culture were crosslinked using $37 \%$ formaldehyde in a final concentration at $1 \%$. Crosslinking was performed by inverting the culture containing tube 10 times at room temperature. In the next step, cells were pelleted by centrifugation $(3500 \mathrm{~g}$, $10 \mathrm{~min}$ ) and washed twice in cold PBS. The lysis of the cells and immunoprecipitation was performed using the $\mu$ MACS $^{\mathrm{TM}}$ His Isolation Kit (Miltenyi Biotec). The pellet was re-suspended in $1 \mathrm{ml}$ of lysis buffer. $100 \mu \mathrm{l}$ lysozyme $(10 \mathrm{mg} / \mathrm{ml})$ were added and incubated for $30 \mathrm{~min}$ on ice. Subsequently $10 \mu \mathrm{l}$ 4-(2-aminoethyl)-benzensulfonylfluorid (AEBSF) $(100 \mathrm{mM})$ and $10 \mu \mathrm{l}$ RNAseA $(10 \mathrm{mg} / \mathrm{ml})$ were added and the sample was incubated another $30 \mathrm{~min}$ on ice. After cell lysis the DNA was sheared on ice by sonication using the Branson Digital Sonifier UNITS Models S-250D. The samples were sonified for $30 \mathrm{sec}$ at $20 \%$ power and incubated on ice for one minute. Sonication was repeated four times. $900 \mu \mathrm{l}$ of the sample were stored at $-80^{\circ} \mathrm{C}$. The remaining $100 \mu \mathrm{l}$ were used for determining the sharing rate and incubated with $10 \mu$ l proteinase $\mathrm{K}$ (10 $\mathrm{mg} / \mathrm{ml}$ ) and $345 \mu \mathrm{l} \mathrm{H}_{2} \mathrm{O}_{\mathrm{dd}}$ at $65^{\circ} \mathrm{C}$ overnight to reverse crosslinking. The DNA was cleaned up using the GeneJet PCR purification Kit (Thermo Fisher) and run on a 1.5\% agarose gel. The sharing range was determined to range from $200 \mathrm{bp}$ to $1000 \mathrm{bp}$ with an average size of DNA fragments around $500 \mathrm{bp}$. The immunoprecipitation was carried out using the $\mu$ MACS Anti-His MicroBeads to isolate his-tagged PdhR protein from the sample following the manufactures' instructions. The $900 \mu \mathrm{l}$ sample was thawed on ice and incubated with the magnetic beads, loaded onto the column and washed with buffers supplemented with the kit. The native DNA-protein-MicroBead complex was eluted in $100 \mu \mathrm{l}$ TE buffer. The isolation of his-tagged PdhR protein was checked by analyzing $5 \mu$ of the elution fraction by SDS-PAGE and Western blot analysis. Crosslinking was reversed by incubation of the remaining $95 \mu \mathrm{l}$ of the elution fraction at $65^{\circ} \mathrm{C}$ overnight. $1 \mu \mathrm{l}$ of proteinase $\mathrm{K}$ was added and the sample incubated at $45^{\circ} \mathrm{C}$ for two hours. In the next steps the DNA was separated from proteins and MicroBeads by a phenol/chloroform extraction. $0.5 \mathrm{ml} \mathrm{phenol/chloroform} \mathrm{was} \mathrm{added} \mathrm{and} \mathrm{the} \mathrm{sample}$ mixed for $5 \mathrm{~min}$. After one minute of incubation without mixing, the sample was mixed again for $2 \mathrm{~min}$. The last two steps were repeated three times. Phase separation was carried out by a centrifugation step at $13000 \mathrm{rpm}$ for 5 min. The DNA containing phase was transferred into a new cup and $1 / 10$ volume of Na-Acetate $(3 \mathrm{M}, \mathrm{pH} 6)$ and $1 \mathrm{ml}$ ethanol were added. The sample was placed at $-20^{\circ} \mathrm{C}$ overnight, centrifuged (13000 rpm, $\left.30 \mathrm{~min}, 4^{\circ} \mathrm{C}\right)$, and washed in $80 \%$ ethanol. The pellet was air-dried and resuspended in $100 \mu \mathrm{T} \mathrm{TE}$.

\section{Quantitative PCR}

Quantitative PCR was performed using the iCycler Thermal Cycler from BioRad. The qPCR was run in 25 $\mu \mathrm{l}$ reactions containing $12.5 \mu \mathrm{l}$ Maxima ${ }^{\mathrm{TM}}$ SYBR Green qPCR Master Mix (Thermo Fisher), $2.5 \mu \mathrm{l}$ primer+ and $2.5 \mu \mathrm{l}$ primer- $(10 \mathrm{pmol} / \mu \mathrm{l}), 5.5 \mu \mathrm{l} \mathrm{H}_{2} \mathrm{O}_{\mathrm{dd}}$ and $2 \mu \mathrm{l}$ of the DNA which was co-precipitated, purified and concentrated. One DNA sample was used as template in six PCR reactions with each of the six primer pairs to compare the relative amount of PCR product. The primer pairs were chosen that they surround the putative binding site and give products of a length between 200 and 300 bps. The primers were checked in a standard endpoint PCR that they only amplify one product of the desired length. An annealing temperature of $51^{\circ} \mathrm{C}$ was determined to be suitable for all primer pairs. Therefore the reaction with all primer pairs could be run in the same qPCR. The primers are listed in Table 3. The results were analyzed with the qbase ${ }^{\text {PLUS }}$ software (Biogazelle).

\section{Purification of his-tagged PdhR}

His-tagged PdhR was produced and purified as described earlier [7]. 
Table 3 Primer pairs for qPCR

\begin{tabular}{|c|c|c|}
\hline Binding site & Sequence $5^{\prime}-3^{\prime}$ & \\
\hline \multirow[t]{2}{*}{$\mathrm{PdhR}_{\mathrm{op}}$} & CACAGTTTCATGATTTC & + \\
\hline & GAGAGTGCCTTCGAG & - \\
\hline \multirow[t]{2}{*}{$\mathrm{GlcD}_{\mathrm{op}}$} & CGGACCTCGTGCACAG & + \\
\hline & GTGCCATCAGTACCG & - \\
\hline \multirow[t]{2}{*}{$\mathrm{MraZ}_{\mathrm{op}}$} & AACGGTGATGACGATG & + \\
\hline & GGTAGGCACTGATAAG & - \\
\hline \multirow[t]{2}{*}{ MetBop } & GTGTAATGCACCTGTC & + \\
\hline & AACCCGCTACGCACTG & - \\
\hline \multirow[t]{2}{*}{$Y_{n f M}$} & ATGCAGCTCTTCCGC & + \\
\hline & TTCTCAGTGTCGCTTG & - \\
\hline \multirow[t]{2}{*}{$\mathrm{Pts}_{\mathrm{op}}$} & GTCGGTAAATCGCTGATGCTGCC & + \\
\hline & CAACAACTGCGGCCAGCGC & - \\
\hline
\end{tabular}

\section{Gel shift assays}

Assays were carried out as described previously [7]. All DNA probes were generated by annealing equimolar amount of fluorescence labeled primers (Thermo Fisher Scientific) of the known or predicted PdhR binding sites. The primers are listed in Table 4; binding regions are marked in grey.

\section{Bioinformatics procedures}

Large-scale analysis of gene expression data from $M^{3 D}$

To elucidate further targets of PdhR we used data from 907 microarray experiments stored in $\mathrm{M}^{3 D}$ [8]. We used the implementation of the context-likelihood of relatedness algorithm [5] provided in the R-package DTInfer [7] to determine potential targets of PdhR. In short, we computed the mutual information between the expression of $p d h R$ and each gene of $E$. coli across the 907 microarray experiments. The significance of each of the mutual information values was estimated by computation of a $\mathrm{z}$-score. This $\mathrm{z}$-score is the square root of the sum of squares of two scores: the $\mathrm{z}^{1}$ and the $\mathrm{z}^{2}$-score. For the mutual information value $I(i, j)$ between the expression vectors of gene $i$ and gene $j, \mathrm{z}^{1}$ corresponds to the relative position of $I(i$, $j)$ in the distribution of all mutual information values involving gene $i$ and $\mathrm{z}^{2}$ to the relative position of $I(i, j)$ in the distribution of all mutual information values involving gene $j$. Since we were interested in regulatory targets of PdhR we subsequently discarded z-scores for all interactions not involving PdhR. By sorting genes according to their z-scores, we obtained a ranking of genes according to their likelihood to be regulated by PdhR. The z-scores of regulatory interactions involving PdhR are given in Additional File 1.

\section{Analysis of expression data from pdhR knockout and overexpressing strains}

To provide an independent line of evidence, we determined the gene expression of four different strains of $E$. coli on three different media. The four strains corresponded to E. coli LJ110, a pdhR knockout mutant (LJ110 $\Delta p d h R$ ), the parental strain carrying an empty plasmid (LJ110/pTM30) and the parental strain overproducing PdhR (LJ110/pTM30PdhRhis). These strains were cultivated on Luria-Bertani broth (LB), standard phosphate minimal medium supplemented with acetate and standard phosphate minimal medium supplemented with pyruvate. We obtained an overall 24 microarray experiments from two biological replicates of each of these cultivations as described above.

To analyse the quality of the microarray data, the raw gene-expression data were quantil-normalized using the package 'preprocessCore' of the Bioconductor Software [30]. The probes with low signal intensity were discarded for further analysis. In order to exclude samples that are not clearly attributable to their culture condition, the following quality check was performed. The high dimensional space of the gene expression data was mapped to a two- or three-dimensional space using the nonlinear Sammon projection method [31] implemented in the $\mathrm{R}$ package 'MASS' [32]. To detect possible outliers in the set of samples a model-based clustering approach using the $\mathrm{R}$ package mclust [33] was performed. This analysis identified three of the 24 microarrays that were not clearly attributable to culture conditions and were thus discarded from the subsequent analysis. To detect genes that were particularly affected by overexpression and knockout of $p d h R$, we determined for each gene $i$ in each medium an average overexpression $o_{i}$ and an average knockout score $k_{i}$ as follows. Overexpression scores were

Table 4 Fluorescence labeled primers for gel shift assays

\begin{tabular}{|c|c|c|}
\hline Binding site & Sequence $5^{\prime}-3^{\prime}$ & Label \\
\hline \multirow[t]{2}{*}{$\mathrm{PdhR}_{\mathrm{op}}$} & GCCGAAGTCAATTGGTCTTACCAATTTCATGTCTGTG & 5'DY682 \\
\hline & CACAGACATGAAATTGGAAGACCAATTGACTTCGGC & $5^{\prime} \mathrm{DY} 782$ \\
\hline \multirow[t]{2}{*}{$\mathrm{GlcD}_{\mathrm{op}}$} & CTATCTCTITAGCTACCGGTCAGACCATTTTTTITCCAGCTCT & 5'DY682 \\
\hline & AGAGCTGGAAAAAAAATGGTCTGACCGGTAGCTAAAGAGATAG & 5'DY782 \\
\hline \multirow[t]{2}{*}{$\mathrm{MraZ}_{\mathrm{op}}$} & TCGGTATGCCTTGTGACTGGCTTGACAAGCTTTTCCTCAGCTCC & 5'DY682 \\
\hline & GGAGCTGAGGAAAAGCTTGTCAAGCCAGTCACAAGGCATACCGA & 5'DY782 \\
\hline \multirow[t]{2}{*}{ MetB $_{\text {op }}$} & AACGGCTATTTGGGATTTGCTCAATCTATACGCAAAGAAGTTT & 5'DY682 \\
\hline & AAACTTCTITGCGTATAGATTGAGCAAATCCCAAATAGCCGTT & $5^{\prime} \mathrm{DY} 782$ \\
\hline
\end{tabular}


determined by subtracting the log expression values of the strain carrying the empty plasmid from the overexpression strain. Knockout scores were obtained by subtracting the log expression value of the parental strain from the knockout strain. Due to the three discarded microarray experiments we thus obtained five knockout and four overexpression scores for each gene. For each gene the average knockout and average overexpression score was determined as average over the knockout and overexpression scores, respectively. Finally, we obtained a regulatory score over all microarrays by subtracting, for each gene, the overexpression score from the knockout score. Since PdhR is known to repress the transcription of most of its targets, we expect known targets of PdhR to have a high regulatory score in our experiments. The regulatory scores of all genes are given in Additional File 1.

\section{Identification of candidates for experimental validation}

To identify potential candidates for experimental validation of potential interactions we determined for each gene of $E$. coli whether we could identify a putative phylogenetically conserved transcription factor binding site of PdhR in its upstream region as described previously [7]. In brief, we aligned known binding sites of PdhR using the R-package cosmo [34] with the promoter region of each gene. If we thus identified a DNA sequence that resembled known binding sites of PdhR, we checked whether the corresponding region coincides with a part of the promoter known to be phylogenetically conserved upstream of genes in ten proteobacterial genomes $[35,36]$. We identified potential binding sites of PdhR in the promoter regions of 363 operons containing 642 genes. We ranked the genes in this list independently according to the $\mathrm{z}$-score of a regulation by PdhR and according to the regulatory score obtained from our own microarray experiments. The top-ranking 20 candidates in either of both lists are displayed in Table 2.

\section{Comparison of gene-expression between acetate and pyruvate grown cultures}

Since no transporter for pyruvate is known in E. coli to date, we aimed to identify the corresponding gene(s) by comparison of gene-expression between acetate and pyruvate grown cultures. Thus, we computed average foldchanges between acetate and pyruvate grown cultures. The gene with the strongest overexpression possessing a putative phylogenetically conserved binding site of PdhR was $y n f M$. This gene encodes a transporter belonging to the major facilatory superfamily of transporters with yet unknown function. Moreover, ynfM showed a high regulatory score in the comparison of PdhR-knockout and overexpression strains (Additional File 1). These results led us to hypothesize that $y n f M$ is a pyruvate transporter in E. coli. However, complementation studies in a mutant strain that does not grow on pyruvate minimal medium could not confirm these results (data not shown).

\section{Additional material}

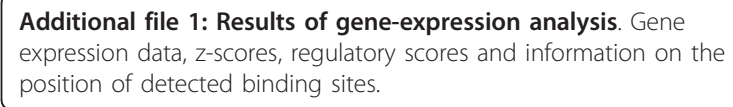

\section{Acknowledgements}

We thank Lucille Schmieding for proof-reading the manuscript. Financial support from the German Ministry of Education and Research (BMBF) within the Jena Centre of Bioinformatics and the FORSYS-Partner program (grant FKZ 0315285) is gratefully acknowledged.

\section{Author details}

${ }^{1}$ Department of Genetics, University of Osnabrück, Barbarastraße 11, D-49076 Osnabrück, Germany. ${ }^{2}$ Helmholtz Centre for Infection Research,

Inhoffenstraße 7, D-38124 Braunschweig, Germany. ${ }^{3}$ Institute of Technical Chemistry - Life Science, Leibniz University of Hannover, Callinstraße 5, D30167 Hannover, Germany. ${ }^{4}$ Systems Biology/Bioinformatics Group, Leibniz Institute for Natural Product Research and Infection Biology - Hans Knöll Institute, D-07745 Jena, Germany. ${ }^{5}$ Research Group Genome Analytics, Helmholtz Centre for Infection Research, Inhoffenstraße 7, D-38124 Braunschweig, Germany. ${ }^{6}$ Department of Bioinformatics, School of Biology and Pharmaceutics, Ernst-Abbe-Platz 2, Friedrich Schiller University of Jena, D-07743 Jena, Germany. ${ }^{7}$ Research Group Theoretical Systems Biology, School of Biology and Pharmaceutics, Leutragraben 1, Friedrich Schiller University of Jena, D-07743 Jena, Germany.

\section{Authors' contributions}

AKG, ÖK and RoG conducted the experiments. CK, WSH, ÖK and RoG analyzed the microarray data. CK, KJ, SS, UR and ReG designed research. AKG, KJ, CK, ÖK and WSH wrote the paper. All authors read and approved the final manuscript.

Received: 11 August 2011 Accepted: 14 December 2011

Published: 14 December 2011

\section{References}

1. Quail MA, Guest JR: Purification, characterization and mode of action of PdhR, the transcriptional repressor of the pdhR-aceEF-Ipd operon of Escherichia coli. Mol Microbiol 1995, 15(3):519-529.

2. Quail MA, Haydon DJ, Guest JR: The pdhR-aceEF-Ipd operon of Escherichia coli expresses the pyruvate dehydrogenase complex. Mol Microbiol 1994, 12(1):95-104.

3. Haydon DJ, Guest JR: A new family of bacterial regulatory proteins. FEMS Microbiol Lett 1991, 63(2-3):291-295.

4. Ogasawara H, Ishida Y, Yamada K, Yamamoto K, Ishihama A: PdhR (pyruvate dehydrogenase complex regulator) controls the respiratory electron transport system in Escherichia coli. J Bacteriol 2007, 189(15):5534-5541.

5. Faith JJ, Hayete B, Thaden JT, Mogno I, Wierzbowski J, Cottarel G, Kasif S, Collins JJ, Gardner TS: Large-scale mapping and validation of Escherichia coli transcriptional regulation from a compendium of expression profiles. PLOS Biol 2007, 5(1):e8.

6. Blankenhorn D, Phillips J, Slonczewski JL: Acid- and base-induced proteins during aerobic and anaerobic growth of Escherichia coli revealed by two-dimensional gel electrophoresis. J Bacteriol 1999, 181(7):2209-2216.

7. Kaleta C, Göhler A, Schuster S, Jahreis K, Guthke R, Nikolajewa S: Integrative inference of gene-regulatory networks in Escherichia coli using information theoretic concepts and sequence analysis. BMC Syst Bio/ 2010, 4:116.

8. Faith JJ, Driscoll ME, Fusaro VA, Cosgrove EJ, Hayete B, Juhn FS, Schneider SJ, Gardner TS: Many Microbe Microarrays Database: uniformly normalized Affymetrix compendia with structured experimental metadata. Nucleic Acids Res 2008, , 36 Database: D866-870. 
9. Freyre-Gonzalez JA, Alonso-Pavon JA, Trevino-Quintanilla LG, ColladoVides J: Functional architecture of Escherichia coli: new insights provided by a natural decomposition approach. Genome Biol 2008, 9(10):R154.

10. Vicente M, Gomez MJ, Ayala JA: Regulation of transcription of cell division genes in the Escherichia coli dcw cluster. Cell Mol Life Sci 1998, 54(4):317-324.

11. Pao SS, Paulsen IT, Saier MH Jr: Major facilitator superfamily. Microbiol Mol Biol Rev 1998, 62(1):1-34.

12. Janga SC, Salgado H, Martinez-Antonio A: Transcriptional regulation shapes the organization of genes on bacterial chromosomes. Nucleic Acids Res 2009, 37(11):3680-3688.

13. Pellicer MT, Fernandez C, Badia J, Aguilar J, Lin EC, Baldom L: Crossinduction of $g / c$ and ace operons of Escherichia coli attributable to pathway intersection. Characterization of the glc promoter. J Biol Chem 1999, 274(3):1745-1752.

14. Kornberg HL, Krebs HA: Synthesis of cell constituents from C2-units by a modified tricarboxylic acid cycle. Nature 1957, 179(4568):988-991.

15. Kornberg HL, Lascelles J: The formation of isocitratase by the Athiorhodaceae. J Gen Microbiol 1960, 23:511-517.

16. Kornberg HL, Phizackerley PJ, Sadler JR: The metabolism of C2 compounds in micro-organisms. 5. Biosynthesis of cell materials from acetate in Escherichia coli. Biochem J 1960, 77:438-445

17. Chao YP, Patnaik R, Roof WD, Young RF, Liao JC: Control of gluconeogenic growth by pps and pck in Escherichia coli. J Bacteriol 1993, 175(21):6939-6944

18. d'Ari R: The SOS system. Biochimie 1985, 67(3-4):343-347.

19. Giese KC, Michalowski CB, Little JW: RecA-dependent cleavage of LexA dimers. J Mol Biol 2008, 377(1):148-161.

20. Bi EF, Lutkenhaus J: FtsZ ring structure associated with division in Escherichia coli. Nature 1991, 354(6349):161-164.

21. Vicente M, Kushner SR, Garrido T, Aldea M: The role of the 'gearbox' in the transcription of essential genes. Mol Microbiol 1991, 5(9):2085-2091.

22. Kotte O, Zaugg JB, Heinemann M: Bacterial adaptation through distributed sensing of metabolic fluxes. Mol Syst Biol 2010, 6:355.

23. Zeppenfeld T, Larisch C, Lengeler JW, Jahreis K: Glucose transporter mutants of Escherichia coli K-12 with changes in substrate recognition of IICB(Glc) and induction behavior of the ptsG gene. J Bacteriol 2000, 182(16):4443-4452

24. Tanaka S, Lerner SA, Lin EC: Replacement of a phosphoenolpyruvatedependent phosphotransferase by a nicotinamide adenine dinucleotidelinked dehydrogenase for the utilization of mannitol. J Bacteriol 1967, 93(2):642-648.

25. Morrison TB, Parkinson JS: Liberation of an interaction domain from the phosphotransfer region of CheA, a signaling kinase of Escherichia coli. Proc Natl Acad Sci USA 1994, 91(12):5485-5489.

26. Yanisch-Perron C, Vieira J, Messing J: Improved M13 phage cloning vectors and host strains: nucleotide sequences of the M13mp18 and pUC19 vectors. Gene 1985, 33(1):103-119.

27. Datsenko KA, Wanner BL: One-step inactivation of chromosomal genes in Escherichia coli K-12 using PCR products. Proc Natl Acad Sci USA 2000, 97(12):6640-6645.

28. Arber W: Transduction of chromosomal genes and episomes in Escherichia coli. Virology 1960, 11:273-288.

29. Lengeler J: Studies on the glucose effect in the synthesis of the galactose enzyme of Escherichia coli. Z Vererbungs/ 1966, 98(3):203-229.

30. Gentleman RC, Carey VJ, Bates DM, Bolstad B, Dettling M, Dudoit S, Ellis B, Gautier L, Ge Y, Gentry J, et al: Bioconductor: open software development for computational biology and bioinformatics. Genome Biol 2004, 5(10): R80.

31. Sammon JW: A nonlinear mapping for data structure analysis. IEEE Transactions on Computers 1969, 18:401-409.

32. Venables WN, Ripley BD: Modern Applied Statistics with S. New York: Springer; 42002.

33. Fraley C, Raftery AE: MCLUST Version 3 for R: Normal Mixture Modeling and Model-based Clustering. Technical Report 504 Department of Statistics, University of Washington; 2006.

34. Bembom O, Keles S, van der Laan MJ: Supervised detection of conserved motifs in DNA sequences with cosmo. Stat Appl Genet Mol Biol 2007, 6 : Article8.
35. McCue L, Thompson W, Carmack C, Ryan MP, Liu JS, Derbyshire V, Lawrence CE: Phylogenetic footprinting of transcription factor binding sites in proteobacterial genomes. Nucleic Acids Res 2001, 29(3):774-782.

36. McCue LA, Thompson W, Carmack CS, Lawrence CE: Factors influencing the identification of transcription factor binding sites by cross-species comparison. Genome Res 2002, 12(10):1523-1532.

37. Keseler IM, Collado-Vides J, Santos-Zavaleta A, Peralta-Gil M, Gama-Castro S, Muniz-Rascado L, Bonavides-Martinez C, Paley S, Krummenacker M, Altman T, et al: EcoCyc: a comprehensive database of Escherichia coli biology. Nucleic Acids Res 2011, , 39 Database: D583-590.

doi:10.1186/1752-0509-5-197

Cite this article as: Göhler et al:: More than just a metabolic regulator elucidation and validation of new targets of PdhR in Escherichia coli. BMC Systems Biology 2011 5:197.

\section{Submit your next manuscript to BioMed Central and take full advantage of:}

- Convenient online submission

- Thorough peer review

- No space constraints or color figure charges

- Immediate publication on acceptance

- Inclusion in PubMed, CAS, Scopus and Google Scholar

- Research which is freely available for redistribution

Submit your manuscript at www.biomedcentral.com/submit
Biomed Central 